\title{
ELETRORRETINOGRAFIA POR PADRÃO REVERSO EM PACIENTES COM ENXAQUECA SEM AURA
}

\author{
PEDRO F. MOREIRA FILHO*, ADALMIR M. DANTAS*
}

\begin{abstract}
RESUMO - Foram submetidos a eletrorretinografia por padrão reverso (PERG) 17 pacientes com o diagnóstico de enxaqueca sem aura através dos critérios estabelecidos pela Sociedade Internacional de Cefaléia. O estímulo usado em nossos enfermos foi o estruturado produzido por aparelho de televisão de 20 polegadas. A frequência foi de l estímulo por segundo e o total de estímulos somados foi de 512 . Constatamos as latências das ondas $P_{s o}$ e $\mathrm{N}_{\text {y5 }}$ dentro dos limites de normalidade em todos os nossos casos, a exceção de um em que no olho esquerdo nāo houve ondas detectadas. Será necessário maior número de exames para que no futuro possamos obter dados que nos ajudem a entender a fisiopatologia da enxaqueca.
\end{abstract}

PALAVRAS-CHAVE: enxaqueca, migrânea, eletrorretinografia por padrão reverso.

\section{Pattern reversal electroretinogram in migraine patients without aura}

SUMMARY - Seventeen patients with migraine without aura were investigated: the age was between 16 and 39 years, 6 were men and 11 women. The diagnosis of migraine was made according to the classification proposed by the International Headache Society. The method of electroretinogram was performed with pattern reversal (PERG), with monocular stimulation. The stimulation was performed with pattern reversal with $4 \times 4 \mathrm{~cm}$ black and white squared screen placed one meter from nasion at stimulus frequency $1 / s ; 512$ individual trials were analysed. The PERG did not show a significant increase in the value of the $P_{50}$ and $N_{95}$ latency in our cases. Specifically in one of our cases no wave was detected in the left eye.

KEY WORDS: migraine, pattern reversal, electroretinogram.

Não é exagero afirmar que a cefaléia tenha a idade da história da humanidade e que, pelo menos a enxaqueca, seja tão velha quanto a própria medicina. Entretanto, a incidência e a fisiopatogenia da migrânea permanecem obscuras. Spierings ${ }^{17}$ relata que, num levantamento populacional nos Estados Unidos, aproximadamente $9 \%$ dos homens, $15 \%$ das mulheres e 3 a $4 \%$ das crianças padecem de enxaqueca. Com esses dados, verificamos que é assustador o número de pessoas que sofrem deste tipo específico de cefaléia denominado enxaqueca. Em nosso meio há uma indiscutível carência de dados estatísticos a este respeito. Já no que diz respeito à fisiopatogenia, as teorias mais aceitas atualmente são: a) teoria vasculars; b) teoria da oligoemia alastrante ${ }^{10}$; e c) teoria da depressão alastrante de Leão ${ }^{11,12}$. Todas elas têm defensores e críticos, não havendo consenso universal a este respeito. Hipócrates, em 400 a.C. já fazia menção aos distúrbios visuais que precedem a crise de enxaqueca ${ }^{6}$. Estas manifestações visuais são frequentes em pacientes migranosos sendo comum a visualização de escotomas cintilantes, a fotofobia e hemianopsias. Sendo a retina uma miniatura do encéfalo, podemos considerá-la um modelo acessível para a avaliação da atividade do sistema nervoso central em pacientes com enxaqueca ${ }^{3}$.

* Departamento de Medicina Clínica (Neurologia) do Hospital Universitário Antônio Pedro da Universidade Federal Fluminense. Aceite: 5-maio-1995.

Dr. Pedro Ferreira Moreira Filho - Av. $\mathrm{N}^{2} \mathrm{Sr}$ de Copacabana 540/606 - 22020-000 Rio de Janeiro RJ - Brasil. 
Com a disponibilidade de um grande número de pacientes migranosos e com excelente serviço de eletrofisiologia ocular é que nos dispusemos a submeter esses enfermos a eletrorretinografia por padrão reverso. Este estudo partiu do interesse em avaliar a retina dos enxaquecosos sem aura através da mediçāo das latências de $\mathrm{P}_{50}$ e $\mathrm{N}_{95}{ }^{1,4}$ na tentativa de obter algum subsídio que pudesse colaborar no melhor entendimento da fisiopatogenia desta afecção. Na literatura estrangeira, praticamente inexistem artigos que abordem este assunto, sendo este estudo pioneiro em nosso meio.

\section{MÉTOdOS}

Foram submetidos a eletrorretinografia por padrão reverso (PERG) 17 pacientes com o diagnóstico de enxaqueca sem aura, obedecendo aos critérios estabelecidos pela Sociedade Intemacional de Cefaléia'. Desses 17 enfermos, 11 cram do sexo feminino e 6 do masculino, com idade de 16 a 39 anos e com tempo de sintomatologia que variava entre 1 a 19 anos (Tabela 1). Todos os pacientes apresentavam exame neurológico normal. Os enfermos foram avaliados oftalmologicamente e aqueles que necessitavam de lentes corretivas só se submeteram ao PERG após esta correção.

Os pacientes que estavam em uso de medicação profilática para migrânea, foram orientados de modo que 7 dias antes do exame (PERG) estes fármacos fossem suspensos. O uso de analgésicos, derivados da ergotamina e benzodiazepínicos só foi permitido quando utilizados até 72 horas antes do exame.

O estímulo usado em nossos 17 pacientes foi o estruturado ou de natureza geométrica, produzido por aparelho de televisão de 20 polegadas. Utilizamos o padrão reverso xadrez em preto e branco.Os quadriláteros de $4 \times 4 \mathrm{~cm}$ usados como estimuladores, correspondem a ângulo visual de cerca de $30^{\circ}$, formando o desenho de um tabuleiro de xadrez ou damas. A luminância permaneceu constante. A frequência foi de 1 segundo em campo cheio, isto é, em toda a tela do aparelho de televisāo. O total de estímulos somados foi de 512.

Precedia o exame a administração de 1 gota de cloridrato de proximetacaína a 0,5\% (anestésico tópco) em cada olho. Três cletrodos foram usados para obter o PERG. O eletrodo ativo de platina, especialmente desenhando, enganchado sobre a pálpebra inferior do olho a ser examinado. O eletrodo de referência (de contato) foi posicionado na regiāo temporal contralateral e o eletrodo terra, também de contato, na regiāo frontal.

Tabela 1. Sexo, idade e tempo de evolução da enxaqueca sem aura dos 17 pacientes estudados.

\begin{tabular}{cccc}
\hline Paciente & Sexo & Idade & $\begin{array}{c}\text { Tempo de evolução } \\
\text { aproximado (anos) }\end{array}$ \\
\hline 1 & M & 38 & 18 \\
2 & F & 36 & 19 \\
3 & F & 29 & 7 \\
4 & M & 24 & 5 \\
5 & F & 18 & 2 \\
6 & F & 17 & 3 \\
7 & M & 18 & 3 \\
8 & F & 38 & 18 \\
9 & F & 27 & 10 \\
10 & F & 28 & 8 \\
11 & F & 19 & 1 \\
12 & M & 33 & 11 \\
13 & F & 21 & 4 \\
14 & F & 39 & 6 \\
15 & F & 21 & 2 \\
16 & M & 16 & 13 \\
17 & M & 39 & 2 \\
\hline
\end{tabular}


Tabela 2. Latência da onda $P_{\text {so }}(m s)$ em 17 pacientes com enxaquecu sem aura.

\begin{tabular}{|c|c|c|}
\hline Paciente & OD & $\mathrm{OE}$ \\
\hline 1 & 50 & nd \\
\hline 2 & 50 & 50 \\
\hline 3 & 50 & 55 \\
\hline 4 & 50 & 50 \\
\hline 5 & 50 & 50 \\
\hline 6 & 50 & 55 \\
\hline 7 & 50 & 55 \\
\hline 8 & 55 & 50 \\
\hline 9 & 55 & 50 \\
\hline 10 & 55 & 50 \\
\hline II & 50 & 50 \\
\hline 12 & 50 & 55 \\
\hline 13 & 50 & 50 \\
\hline 14 & 50 & 50 \\
\hline 15 & 47 & 55 \\
\hline 16 & 50 & 55 \\
\hline 17 & 50 & 50 \\
\hline
\end{tabular}

ms, milisegundos; OD, olho direito; OE, olho esquerdo; nd, nāo detectável..
Tabela 3. Latência da onda $N_{y 5}$ (ms) em 17 pacientes com enxaqueca sem aura.

\begin{tabular}{|c|c|c|}
\hline Paciente & OD & $\mathrm{OE}$ \\
\hline 1 & 90 & nd \\
\hline 2 & 95 & 95 \\
\hline 3 & 100 & 100 \\
\hline 4 & 95 & 95 \\
\hline 5 & 95 & 95 \\
\hline 6 & 95 & 95 \\
\hline 7 & 95 & 100 \\
\hline 8 & 100 & 95 \\
\hline 9 & 105 & 100 \\
\hline 10 & 95 & 95 \\
\hline 11 & 90 & 95 \\
\hline 12 & 95 & 100 \\
\hline 13 & 90 & 95 \\
\hline 14 & 100 & 95 \\
\hline 15 & 90 & 105 \\
\hline 16 & 90 & 100 \\
\hline 17 & 95 & 100 \\
\hline
\end{tabular}

ms, milisegundos; OD, olho direito; $\mathrm{OE}$, olho esquerdo; nd, não detectável.

Em ambos os eletrodos de contato utilizou-se uma pasta eletrolítica.

O exame foi realizado em local sem ruídos. O paciente permaneceu sentado a $I$ metro do aparelho de televisão que foi especialmente adaptado para a estimulaçāo através do padrão xadrez reverso. Esta era a única fonte luminosa do ambiente. A estimulaçāo toi do tipo monocular. Inicialmente cobria-se o olho esquerdo e se registrava o traçado do olho direito, procedendo-se posteriormente a mesma operação com o olho esquerdo.

O grupo controle foi constituído de igual número de pacientes (17) com diagnóstico de enxaqueca sem aura através dos critérios da Sociedade Intemacional de Cefaléia. Desses 17 enfermos, 14 eram do sexo feminino e 3 do masculino, com idade de 19 a 54 anos. Nenhum dos pacientes estava em uso de qualquer medicação há pelo menos 5 dias antes de serem submetidos à eletrorretinografia por flash (ERG-F).

No primeiro tempo, dilatou-se a pupila de ambos os olhos dos enfermos com tropicamida a $1 \%$, usandose a seguir colírio anestésico (cloridrato de proximetacaína à $0,5 \%$ ). Em um segundo tempo procedeu-se a colocação dos três eletodos. O eletrodo ativo foi colocado na córnea sob a forma de lente de contato, o eletrodo de referência na regiāo supra orbitária e o terra na região frontal.

Em seguida aplicou-se o estímulo luminoso com flash de 20 Joules com o olho adaptado à luz (fotópico) e 15 minutos após fez-se novo estímulo com o olho adaptado ao escuro (escotópico). A estimulação foi do tipo monocular. Inicialmente foi feito o estímulo no ollho direito e depois o mesmo processo foi repetido no olho esquerdo. 


\section{RESULTADOS} amplitudes.

As Tabelas 2 e 3 mostram as latências de $P_{50}$ e $N_{95}$ respectivamente e as Tabelas 4 e 5 , as

As latências das ondas $P_{50}$ do PERG nos 17 enfermos variaram de 47 a $55 \mathrm{~ms}$ (Tabela 2) e das ondas $\mathrm{P}_{4,5}$ de 90 a $105 \mathrm{~ms}$ (Tabela 3). Em ambos os casos tais valores obtidos estāo compreendidos dentro dos limites de normalidade.

As amplitudes das ondas $P_{50}$ do $P E R G$ nos nossos 17 pacientes mostraram grande variabilidade com valores entre 6 a $22 \mu \mathrm{V}$ (Tabela 4). O mesmo fenômeno foi observado nas amplitudes das ondas $\mathrm{N}_{95}$, com variaçöes de 8 a $38 \mu \mathrm{V}$ (tabela 5).

Os valores de latência e amplitude foram verificados $\mathrm{cm}$ todos os nossos enfermos, com exceção do Paciente 1 em cujo olho esquerdo não conseguimos obter nem a onda $P_{50}$ nem a $N_{95}$.

\section{COMENTÁRIOS}

O padrão reverso foi utilizado, em 1988, num estudo de Mariani et al. ${ }^{14}$ empregando o potencial evocado visual (PEV) em enfermos com enxaqueca sem aura. Os resultados obtidos não mostraram diferenças significativas nas latências e nas amplitudes da onda $\mathrm{P}_{10 x\}}$ dos pacientes migranosos quando

Tabela 4. Amplitude da onda $P_{s o}(\mu \mathrm{V})$ em 17 pacientes com enxaqueca sem aura.
Tabela 5. Amplitude da Onda $N_{y 5}(\mu V)$ em 17 pacientes com enxaqueca sem aura.

\begin{tabular}{cccccc}
\hline Paciente & OD & OE & Paciente & OD & OE \\
\hline \hline 1 & 14 & nd & 1 & 24 & nd \\
2 & 6 & 16 & 2 & 10 & 36 \\
3 & 16 & 14 & 3 & 22 & 20 \\
4 & 20 & 22 & 4 & 30 & 38 \\
5 & 16 & 6 & 5 & 28 & 8 \\
6 & 14 & 16 & 6 & 20 & 28 \\
7 & 14 & 16 & 7 & 20 & 30 \\
8 & 8 & 10 & 8 & 12 & 14 \\
9 & 18 & 8 & 9 & 24 & 24 \\
10 & 10 & 8 & 10 & 18 & 14 \\
11 & 28 & 26 & 11 & 34 & 34 \\
12 & 20 & 20 & 12 & 30 & 38 \\
13 & 12 & 10 & 13 & 18 & 10 \\
14 & 14 & 18 & 14 & 22 & 24 \\
15 & 12 & 18 & 15 & 18 & 26 \\
16 & 14 & 14 & 16 & 22 & 22 \\
17 & 10 & 20 & 17 & 12 & 22 \\
\hline
\end{tabular}

$\mu \mathrm{V}$, microvolts; OD, olho direito; OE, olho esquerdo; $\mu \mathrm{V}$, microvolts; $\mathrm{OD}$, olho direito; $\mathrm{OE}$, olho esquerdo; n.d., não detectável. nd, não detectável. 
comparados ao grupo controle. Em 1990, os mesmos autores ${ }^{15}$ obtiveram o potencial evocado visual por padrão reverso (PEV-PR) em 20 pacientes com enxaqueca com aura. Dez desses enfermos também apresentavam crises de migrânea sem aura. Na análise dos resultados, observaram aumento da latência de $P_{100}$ nos pacientes migranosos em relação ao grupo controle. Nas amplitudes, os valores obtidos foram muito variáveis tanto nos pacientes migranosos quanto nos pacientes do grupo controle.

Em nosso meio, Moreira Filho ${ }^{16}$ em 30 pacientes com enxaqueca sem aura submetidos a PEV-PR preto e branco e vermelho e verde, obtve as latências de onda $P_{100}$ normais em 11 enfermos. Em 10 casos as latências de $P_{100}$ estavam aumentadas no PEV-PR preto e branco e vermelho e verde. As latências da onda $P_{110}$ encontravam-se aumentadas somente no PEV-PR vermelho e verde, estando normais no PEV-PR preto e branco em 8 casos. Em 1 caso as ondas não foram detectáveis nem no PEV-PR preto e branco nem no vermelho e verde.

Quanto à eletrorretinografia por padrão reverso (PERG) não existe na bibliografia qualquer estudo realizado com pacientes migranosos. Dantas et al. ${ }^{5}$ atribuem o comprometimento da onda $P_{\text {so }}$ a patologistas da retina e da mácula, enquanto que a onda $\mathrm{N}_{45}$ traduziria prováveis afecçōes do nervo óptico. Desta mesma opinião compartilham Weinstein et al. ${ }^{18}$ e Fishman e Sokol ${ }^{7}$.

$\mathrm{Na}$ análise das latências das ondas $\mathrm{P}_{50}$ e $\mathrm{N}_{95}$ dos nossos 17 pacientes, encontramos valores dentro dos limites de normalidade conforme os critérios de Chiappa ${ }^{4}$.

As amplitudes das ondas $\mathrm{P}_{50}$ e $\mathrm{N}_{45}$ foram de limitada utilidade como instrumento de interpretação, em virtude da grande variação dos resultados obtidos em nossos casos.

Em um dos nossos pacientes (Caso 1), não obtivemos as ondas $\mathrm{P}_{50}$ e $\mathrm{N}_{95}$ durante o estudo no olho esquerdo. Uma das explicações possíveis para tal achado se basearia na teoria da depressão alastrante de Leāo, circunstância na qual os potenciais evocados não são detectáveis. ${ }^{13}$ Contrariando esta hipótese existe o fato de que este paciente (Caso 1) não estava em crise de enxaqueca no momento da realização do PERG. Uma segunda possibilidade seria, como demonstraram Bumgartner e Epstein ${ }^{2}$, a dificuldade que alguns pacientes possuem de, embora olhando para a fonte de estímulo, não conseguirem fixar atenção, motivo pelo qual as ondas não seriam detectáveis.

Concluímos que, apesar de no estudo realizado em nossos 17 pacientes as latências das ondas $\mathrm{P}_{50} \propto \mathrm{N}_{45}$ terem permanecido dentro dos limites da normalidade, o PERG se constitua num promissor método de investigação diagnóstica e terapêutica para pacientes portadores de migrânea.

Estamos convencidos de que estudo semelhante, cujos objetivos fossem ampliados e, de forma mais abrangente, pudesse envol ver número maior de pacientes, que fossem submetidos ao exame tambćm durante as crises, na vigência ou não do uso de drogas anti-enxaquecosas específicas e, comparativamente, fossem analisados os resultados, certamente nos levaria a conclusões mais seguras e definitivas quanto a fisiopatogenia da migrânea, assim como quanto aos mecanismos de ação e a eficácia terapêutica das diversas substâncias utilizadas no tratamento da enxaqueca.

\section{REFERÊNCIAS}

1. Beminger T, Arden GB. The pattern electroretinogram. In Heckenlively JR, Arden GB eds. Principles and practice of clinical electrophysiology of vision. St. Louis: Mosby Year Book, 1991, p. 29 l-300.

2. Bumgartner J, Epstein C. Voluntary alteration of visual evoked potentials. Ann Neurol 1982, 12: 475-478.

3. Celesia GG. Anatomy and physiology of visual evoked potential and electroretinograms. Neurol Clin 1988, 6: 657-679.

4. Chiappa KH. Pattern-shift evoked potentials: methodology. In Evoked potentials in clinical medicine. Ed 2. New York: Raven Press, 1990, p. 37-109.

5. Dantas AM, Yamane R, Camara AG, Poletti S. PERG eletrorretinograma por padrão xadrez. Rev Bras Oftalin 1988, 47: 17-19.

6. Farias da Silva W. Enxaqueca. In Cefaléias: diagnóstico e tratamento. Rio de Janeiro: Medsi, 1989, p. 61-122. 
7. Fishman GA, Sokol S. The electroretinogram in retinal discase. In: Electrophysiologic testing in disorders of the retina, optic nerve, and visual pathway. Singapore: Palace Press, 1990, p. 25-27.

8. Graham JR, Wolff HG. Mechanism of migraine headache and action of ergotamine tartrate. Arch Neurol Psychiat 1938, 39: 737-763.

9. Headache Classification Committee of the International Headache Society. Classification and diagnostic criteria for headache disorders, cranial neuralgias and facial pain. Cephalalgia 1988, 8: 1-96

I0. Lauritzen $M$, Olsen TS, Lassen NA, Paulson OB. Changes in regional cerebral blood flow during the course of classic migraine attacks. Ann Neurol 1983, 13: 633-641.

11. Leão AAP. Spreading depression of activity in the cerebral cortex. J. Neurophysiol 1944, 7: 359-390.

12. Leāo AAP. What is cortical spreading depression? In Amery WK, Wauquier A. The prelude to the migraine attack. London: Ballière Tindal, 1986, p. 129-133.

13. Maranhão PA Filho. Depressão alastrante e a aura da enxaqueca clássica e experiências acerca dos efeitos da glutamina sobre a depressão alastrante cortical. Tese de Mestrado, Universidade Federal do Rio de Janeiro. Rio de Janciro, 1989.

14. Mariani E, Moschini V, Pastorino G, Rizzi F, Severgnini A, Tiengo M. Pattern - reversal visual evoked potentials and EEG correlations in common migraine patients. Headache 1988, 28: 269-27I.

15. Mariani E, Moschini V, Pastorino G, Rizzi F, Severgnini A, Tiengo M. Pattern reversal visual evoked potentials in migraine subjects with visual aura. Headache 1990, 30: 435-438.

16. Moreira PF Filho. Latência da onda $P_{100}$ do potencial evocado visual padrāo reverso em portadores de enxaqueca sem aura. Tese de Doutorado, Universidade Federal do Rio de Janeiro. Rio de Janeiro, 1992.

17. Spierings ELH. The pathogenesis of the migraine aura: an overview. In: Amery WK, Wauquier A. The prelude to the migraine attack. London: Ballière Tindal, 1986, p. 117-128.

18. Wienstein GW, Odom JV, Cavender S. Visually evoked potentials and electroretinography in neurologic evaluation. Neurol Clin 1991, 9: 225-242. 